\title{
Banco de termos para a prática clínica de enfermagem com idosos comunitários
}

\author{
Jorge Wilker Bezerra Clares ${ }^{1}$, Maria Miriam Lima da Nóbrega², Maria Vilaní Cavalcante Guedes ${ }^{3}$, \\ Lúcia de Fátima da Silva ${ }^{4}$, Maria Célia de Freitas ${ }^{5}$
}

\footnotetext{
${ }^{1}$ Enfermeiro, Mestre em Cuidados Clínicos em Saúde. Enfermeiro da Associação das Pioneiras Sociais. São Luís, MA, Brasil. Email: jorgewilker_clares@yahoo.com.br.

${ }^{2}$ Enfermeira, Doutora em Enfermagem. Professora Titular da Universidade Federal da Paraíba. João Pessoa, PB, Brasil. E-mail: miriam@ccs.ufpb.br.

${ }^{3}$ Enfermeira, Doutora em Enfermagem. Professora Adjunto da Universidade Estadual do Ceará. Fortaleza, CE, Brasil. Email: vilani.guedes@globo.com.

${ }^{4}$ Enfermeira, Doutora em Enfermagem. Professora Adjunto da Universidade Estadual do Ceará. Fortaleza, CE, Brasil. Email: lucia.fatima@uece.br.

${ }^{5}$ Enfermeira, Doutora em Enfermagem. Professora Titular da Universidade Estadual do Ceará. Fortaleza, CE, Brasil. E-mail: celfrei@hotmail.com.
}

Recebido: 29/05/2015.

Aceito: 21/03/2016.

Publicado: 30/06/2016.

Como citar esse artigo:

Clares JBW, Nóbrega MML, Guedes MVC, Silva LF, Freitas MC. Banco de termos para a prática clínica de enfermagem com idosos comunitários. Rev. Eletr. Enf. [Internet]. 2016 [acesso em: ___;18:e1167. Disponível em: http://dx.doi.org/10.5216/ree.v18.37759.

\begin{abstract}
RESUMO
Pesquisa metodológica que objetivou construir um banco de termos para a prática clínica de enfermagem com idosos acompanhados pelas equipes da Estratégia Saúde da Família, com base em documentos oficiais sobre idosos publicados no Brasil, e na Classificação Internacional para a Prática de Enfermagem (CIPE ${ }^{\circledR}$ ), e validá-lo com enfermeiros especialistas da área. Foi desenvolvida no período de 2013 a 2014, baseada em análise documental, identificação, extração, normalização, validação e mapeamento cruzado de termos considerados relevantes para a prática clínica. Identificaram-se 332 termos, que foram submetidos ao processo de mapeamento cruzado, o que resultou em 271 constantes e 61 não constantes na $\mathrm{CIPE}^{\circledR}$ versão 2013. Espera-se que o uso desses termos possibilite a unificação da linguagem profissional do enfermeiro e a implementação da sistematização da assistência de enfermagem nesse nível de atenção. Descritores: Idoso; Vocabulário; Terminologia; Enfermagem; Atenção Primária à Saúde.
\end{abstract}

\section{INTRODUÇÃO}

A discussão acerca do envelhecimento humano e do acesso aos serviços de saúde pela população idosa se intensificou nos últimos anos em virtude de seu crescimento rápido e intenso, sobretudo nos países em desenvolvimento, como o Brasil. Apesar de ser considerado um grande avanço, esse processo resulta em maior carga de doenças e mais incapacidades na população, acarretando maior demanda por serviços de saúde. Além disso, as progressivas alterações decorrentes do processo de envelhecimento, associadas ou não a alguma doença primária, provocam diversas modificações funcionais e estruturais de órgãos e tecidos 
que refletem em todo organismo do idoso, com impacto negativo na sua funcionalidade, autonomia e independência ${ }^{(1)}$.

Esse fenômeno desperta interesse para a necessidade de oferecer uma atenção diferenciada que favoreça um cuidado individualizado e contemple as reais demandas desse grupo. Nessa direção, a Atenção Primária à Saúde (APS), por meio da Estratégia Saúde da Família (ESF), constitui-se espaço privilegiado para atenção integral ao idoso, destacando o enfermeiro no cuidado e no planejamento de ações de promoção, prevenção, educação e intervenção, de forma contextualizada com a realidade vivenciada pela população no âmbito familiar e comunitário ${ }^{(2)}$. Para a efetividade de sua prática clínica, os enfermeiros devem desenvolver ações de cuidado sistematizadas, fundamentadas em conhecimento científico e com enfoque nas necessidades do idoso.

Para realizar o cuidado de enfermagem de forma sistematizada tem-se tornado fundamental o uso de sistemas de classificação de enfermagem, dentre os quais se destaca a Classificação Internacional para a Prática de Enfermagem (CIPE ${ }^{\circ}$ ), que tem sua estrutura desenvolvida com base no Modelo de Sete Eixos, contendo termos que devem ser utilizados na estruturação dos diagnósticos, resultados e intervenções de enfermagem ${ }^{(3)}$. Tal classificação favorece a organização do cuidado clínico de enfermagem e o avanço científico, tecnológico e inovador da profissão ${ }^{(4)}$.

Apesar das vantagens dos sistemas de classificação e da CIPE para a prática clínica de enfermagem, verifica-se que seu uso ainda é incipiente pelos enfermeiros da ESF, principalmente em relação ao cuidado ofertado à população idosa. Além disso, a falta de uma linguagem própria da profissão e de registros adequados de sua prática clínica são problemas evidenciados nesse nível de atenção, repercutindo diretamente na qualidade da assistência prestada e no reconhecimento da profissão, uma vez os registros da prática permitem a avaliação e a geração de conhecimentos ${ }^{(5)}$.

Dessa forma, os enfermeiros precisam lançar mão de um vocabulário específico, uma vez que a padronização da linguagem proporcionará à profissão elementos essenciais para descrever e qualificar a sua assistência, melhorar a comunicação entre os profissionais de enfermagem e outros membros da equipe de saúde em diferentes contextos e culturas, garantir maior autonomia e visibilidade ao trabalho do enfermeiro e subsidiar a prestação de cuidado baseado em evidências ${ }^{(6)}$.

Considerando que o Conselho Internacional de Enfermeiras (CIE) necessita coletar e codificar termos utilizados pela Enfermagem em clientes e áreas específicas para a estruturação de subconjuntos terminológicos, definidos como um conjunto de enunciados de diagnósticos, resultados e intervenções de enfermagem para diferentes especialidades de cuidado, o presente estudo teve como objetivo construir um banco de termos para a prática clínica de enfermagem com idosos acompanhados pelas equipes da ESF, com base em documentos oficiais sobre idosos publicados no Brasil, e na CIPE , e validá-lo com enfermeiros especialistas da área.

\section{MÉTODOS}


Trata-se de um estudo metodológico, realizado no período de 2013 a 2014, que teve por base a análise documental, aplicada a documentos oficiais sobre idosos e ao Modelo de Sete Eixos da CIPE versão 2013, para identificação de termos considerados, clínica e culturalmente, relevantes para a prática clínica de enfermagem na APS.

O processo de extração de termos ocorreu durante o ano de 2013 , e foi realizada por um único pesquisador, mediante a adoção de diretrizes que possibilitaram a uniformização das estratégias utilizadas ${ }^{(4)}$. Foram utilizadas as seguintes referências: Política Nacional do Idoso ${ }^{(7)}$, Estatuto do Idoso ${ }^{(8)}$, Política Nacional de Saúde da Pessoa Idosa ${ }^{(9)}$, Caderno de Atenção Primária no. 19 - Envelhecimento e Saúde da Pessoa Idosa ${ }^{(10)}$ e Guia Prático do Cuidador ${ }^{(11)}$.

Esses documentos foram submetidos à leitura exaustiva e posterior extração dos termos, que foram decompostos em termos simples (substantivos, verbos, advérbios e adjetivos), gerando uma listagem de termos, que foi transcrita para fichas terminológicas criadas em uma planilha eletrônica, no Microsoft Office Excel, totalizando, inicialmente, 880 termos. Em seguida, foram excluídos os termos relacionados a procedimentos médicos, processos patológicos e a medicamentos, resultando em 616 termos. Estes passaram por um processo de normalização e uniformização, com retirada de duplicações, correções gráficas e adequações de gênero e número, totalizando 373 termos.

Após a inclusão desses termos em um formulário, iniciou-se a validação por especialistas da área de Enfermagem. Para a escolha do número de especialistas, consideraram-se recomendações propostas por referencial teórico específico ${ }^{(12)}$, que sugere de cinco a dez sujeitos participando desse processo.

Foram utilizados os seguintes critérios para a inclusão de especialistas: enfermeiros que tivessem, no mínimo, a titulação acadêmica de mestre, trabalhassem com processo de enfermagem e com a CIPE ${ }^{\bullet}$ e tivessem como foco a saúde do idoso na assistência, no ensino e/ou na pesquisa.

Para a identificação de enfermeiros especialistas que preenchessem os critérios de inclusão, realizouse uma pesquisa acessando a Plataforma Lattes, do site do Conselho Nacional de Desenvolvimento Científico e Tecnológico (CNPq). Foram enviados convites a 15 enfermeiros que preenchiam os critérios de inclusão, dos quais apenas cinco aceitaram participar do estudo, mediante devolução do formulário encaminhado preenchido e assinatura do Termo de Consentimento Livre e Esclarecido (TCLE), compondo a amostra final.

Ao grupo de peritos foi solicitado que expressassem o grau de concordância ou discordância em relação aos termos identificados para a prática profissional de enfermagem ao idoso acompanhando na ESF. Em caso de discordância, requisitou-se, se possível, sugestões para sua adequação à realidade da prática clínica de enfermagem.

Para o tratamento dos dados coletados na pesquisa, calculou-se o Índice de Concordância (IC) entre os peritos para cada termo por meio da fórmula: $I C=N C /(N C+N D)$, em que $N C=$ número de concordância e $\mathrm{ND}=$ número de discordância ${ }^{(13)}$. Consideraram-se validados os termos que alcançaram um IC $\geq 0,80$.

Os termos validados foram importados das planilhas do Microsoft Office Excel para o programa Microsoft Office Access para a construção de tabela de termos, e foram submetidos ao processo de 
mapeamento cruzado com os termos do Modelo de Sete Eixos da CIPE versão $2013^{(14)}$. Isso resultou na ligação dos termos identificados nos documentos com os termos da CIPE', identificando-se os termos constantes e não constantes nessa terminologia. Após esse processo, os termos constantes e não constantes foram agrupados em ordem alfabética para constituírem o Banco de Termos para a Prática Clínica de Enfermagem ao Idoso na Atenção Primária.

O presente estudo foi apreciado pelo Comitê de Ética em Pesquisa da Universidade Estadual do Ceará, em observância aos aspectos éticos preconizados na Resolução no. 466/12, do Conselho Nacional de Saúde do Ministério da Saúde ${ }^{(15)}$, obtendo protocolo de aprovação no 501.721, CAAE: 18669013.7.0000.5534.

\section{RESULTADOS}

Dos 373 termos submetidos ao processo de validação de conteúdo por enfermeiros especialistas, 332 termos foram considerados validados obtendo uma concordância $\geq 0,80$. Estes termos foram cruzados com os termos da CIPE versão 2013, resultando em 271 termos constantes e 61 termos não constantes nessa classificação. Os 271 termos constantes foram classificados de acordo com o Modelo de Sete Eixos da CIPE , assim distribuídos: 49 ficaram no eixo Ação, oito no eixo Cliente, 138 no eixo Foco, 10 no eixo Julgamento, 29 no eixo Localização, 27 no eixo Meios e 10 no eixo Tempo. Para classificar os 61 termos não constantes, de acordo com os eixos da CIPE Versão 2013, levaram-se em consideração a definição do eixo e sua coerência com o significado dos termos identificados, resultando em: sete termos no eixo Ação; um no eixo Cliente; 29 no eixo Foco; um no eixo Julgamento; oito no eixo Localização; 12 no eixo Meios; e três no Tempo (Quadros $1,2$ e 3$)$

Quadro 1: Termos relevantes para a prática clínica de enfermagem com idosos comunitários, segundo eixos Ação e Cliente da CIPE versão 2013. Fortaleza, CE, Brasil, 2014.

\begin{tabular}{|c|c|c|}
\hline Eixos & Termos constantes na CIPE ${ }^{\oplus}$ & Termos não constantes na CIPE \\
\hline $\begin{array}{c}\text { Ação } \\
\text { (56 termos) }\end{array}$ & $\begin{array}{l}\text { Aconselhar; Ação; Alimentar-se; Alterar; Aplicar; Apoiar; Assegurar; Atender; } \\
\text { Avaliar; Banhar-se; Cateterizar; Colaborar; Consultar; Colocar Roupas; Contatar; } \\
\text { Controlar; Coordenar; Desenvolver; Encaminhar; Encorajar; Estabelecer; } \\
\text { Estimular; Evitar; Explicar; Facilitar; Guiar; Intervir; Investigar; Monitorar; } \\
\text { Notificar; Orientar; Planejar; Preparar; Prescrever; Prevenir; Priorizar; } \\
\text { Promover; Proporcionar; Proteger; Reabilitar; Reforçar; Registrar; Regular; } \\
\text { Restringir; Supervisionar; Transferir; Trocar; Verificar; Vestir-se/Despir-se. }\end{array}$ & $\begin{array}{c}\text { Acompanhar; Assistir TV; Atualizar; } \\
\text { Buscar; Contribuir; Fazer Sozinho; } \\
\text { Programar. }\end{array}$ \\
\hline $\begin{array}{c}\text { Cliente } \\
\text { (09 termos) }\end{array}$ & Adulto; Casal; Cuidador; Família; Grupo; Idoso; Indivíduo; Paciente. & Amigo \\
\hline
\end{tabular}


Quadro 2: Termos relevantes para a prática clínica de enfermagem com idosos comunitários, segundo eixo Foco da CIPE versão 2013. Fortaleza, CE, Brasil, 2014.

\begin{tabular}{|c|c|c|}
\hline Eixos & Termos constantes na CIPE & Termos não constantes na CIPE \\
\hline $\begin{array}{l}\text { Foco } \\
(167 \\
\text { termos) }\end{array}$ & $\begin{array}{l}\text { Abandono; Abuso Sexual; Adaptação; Adesão; Alcoolismo; Alergia; } \\
\text { Angústia; Ansiedade; Apetite; Apoio Emocional; Atenção; Atitude; } \\
\text { Audição; Autocuidado; Autoestima; Autonomia; Banho; Capacidade } \\
\text { olfativa; Capacidade para colocação de roupas; Complicação; } \\
\text { Comportamento; Comportamento agressivo; Comportamento } \\
\text { sexual; Comunicação; Conforto; Confusão; Constipação; Continência } \\
\text { intestinal; Continência urinária; Controle; Cuidado; Deambulação; } \\
\text { Deglutição; Delírio; Demência; Dentição; Depressão; Desconforto; } \\
\text { Desempenho de papel; Desmaio; Diabetes; Diarreia; Dignidade; } \\
\text { Direitos do paciente; Discriminação; Dor; Edema; Efeito adverso da } \\
\text { medicação; Efeito analgésico; Efeito colateral; Enfrentamento; } \\
\text { Equilíbrio; Esgotamento; Esperança; Estresse; Exame físico; } \\
\text { Exercício; Fadiga; Febre; Ferida; Fezes; Flatulência; Fome; Fratura; } \\
\text { Hidratação; Higiene; Hiperglicemia; Hipertensão; Hipertermia; } \\
\text { Hipoglicemia; Hipotensão; Hipotermia; Impactação; Impotência; } \\
\text { Impotência sexual; Incapacidade; Incontinência de urgência; } \\
\text { Incontinência intestinal; Incontinência urinária; Infecção; } \\
\text { Inflamação; Ingestão de alimentos; Ingestão de líquidos; } \\
\text { Insegurança; Insônia; Integridade; Integridade da pele; Isolamento } \\
\text { social; Lazer; Lesão; Ligação afetiva; Luto; Má nutrição; Mastigação; } \\
\text { Medo; Memória; Micção; Mobilidade; Morte; Mudança de posição; } \\
\text { Necessidade; Negligência; Nutrição; Obesidade; Papel da família; } \\
\text { Peso; Polifármacos; Prazer; Pressão sanguínea; Procedimento; } \\
\text { Processo; Prurido; Qualidade de vida; Queda; Realização; Retenção } \\
\text { urinária; Saúde; Sede; Segurança; Sentar; } \\
\text { musculoesquelético; Sobrepeso; Sofrimento; Sono; Suicídio; } \\
\text { Tabagismo; Temperatura; Tolerância à dieta; Tomada de decisão; } \\
\text { Tontura; Tosse; Trauma; Tristeza; Úlcera por pressão; Urina; } \\
\text { Vergonha; Violência; Vômito. }\end{array}$ & 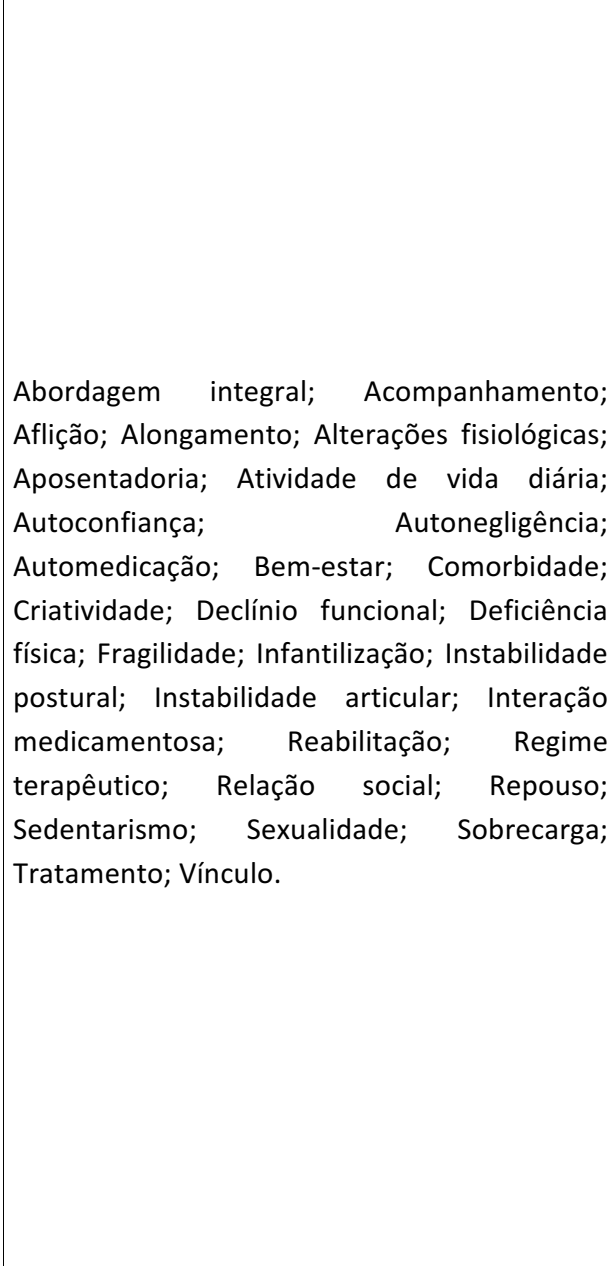 \\
\hline
\end{tabular}

Quadro 3: Termos relevantes para a prática clínica de enfermagem com idosos comunitários, segundo eixos Julgamento, Localização, Meios e Tempo da CIPE versão 2013. Fortaleza, CE, Brasil, 2014.

\begin{tabular}{|c|c|c|}
\hline Eixos & Termos constantes na CIPE & Termos não constantes na CIPE \\
\hline $\begin{array}{l}\text { Julgamento } \\
\qquad(11 \\
\text { termos) }\end{array}$ & $\begin{array}{l}\text { Adequado; Alto; Complexo; Dependência; Eficaz; Estado; Parcial; } \\
\text { Prejudicado; Risco; Total. }\end{array}$ & Assintomático. \\
\hline $\begin{array}{l}\text { Localização } \\
\qquad(37 \\
\text { termos) }\end{array}$ & $\begin{array}{c}\text { Abdome; Ambulatório; Esfíncter anal; Bexiga urinária; Cavidade oral; } \\
\text { Braço; Cabeça; Cabelo; Corpo; Hospital-dia; Intestino; Mama; Músculo; } \\
\text { Olhos; Ombro; Ouvido; Pele; Pênis; Posterior; Próstata; Pulmão; } \\
\text { Períneo; Vagina; Veia; Via intramuscular; Via intravenosa; Via oral; Via } \\
\text { subcutânea; Vizinhança. }\end{array}$ & $\begin{array}{l}\text { Ambiente; Área de abrangência; Casa; } \\
\text { Centro de convivência; Domicílio; Joelho; } \\
\text { Ostomia; Unidade básica de saúde. }\end{array}$ \\
\hline $\begin{array}{l}\text { Meios } \\
\text { (39 } \\
\text { termos) }\end{array}$ & $\begin{array}{l}\text { Alimento; Amputação; Assistente social; Serviço de cuidado domiciliar; } \\
\text { Bengala; Cadeira de rodas; Cama; Ducha; Cirurgia; Curativo de ferida; } \\
\text { Droga; Enema; Equipe interprofissional; Enfermeiro; Fralda; Lentes de } \\
\text { contato; Medicação; Médico; Órtese; Prontuário do paciente; Prótese; } \\
\text { Quimioterapia; Radioterapia; Relaxamento Muscular; Serviço de saúde; } \\
\text { Vacina; Vestuário. }\end{array}$ & $\begin{array}{l}\text { Acompanhante; Agente comunitário de } \\
\text { saúde; Agressor; Ajudante; Andador; } \\
\text { Barras de apoio; Caderneta de saúde da } \\
\text { pessoa idosa; Educação em saúde; Penico; } \\
\text { Preservativo; Sonda; Urinol. }\end{array}$ \\
\hline $\begin{array}{l}\text { Tempo } \\
\text { (13 } \\
\text { termos) }\end{array}$ & $\begin{array}{l}\text { Agudo; Crônico; Dia; Frequência; Hoje; Hospitalização; Idade; } \\
\text { Menopausa; Situação; Visita domiciliária. }\end{array}$ & Horário; Subagudo; Velhice. \\
\hline
\end{tabular}




\section{DISCUSSÃO}

O empenho em padronizar e unificar a linguagem profissional do enfermeiro atende à finalidade da produção do conhecimento da enfermagem, que consiste em compreender as necessidades da população e incorporar novas tecnologias ao cuidado em saúde, contribuindo com a melhoria da prática clínica e consolidação do Sistema Único de Saúde (SUS) ${ }^{(16)}$.

Mediante a análise exploratória e exaustiva dos documentos oficiais sobre idosos publicados no Brasil (Política Nacional do Idoso, Estatuto do Idoso, Política Nacional de Saúde da Pessoa Idosa, Caderno de Atenção Primária no.19, e Guia Prático do Cuidador), foi possível identificar e extrair os termos que, após os processos de normalização, validação e mapeamento cruzado, resultou em 271 termos constantes e 61 termos não constantes na CIPE versão 2013.

A identificação de termos relacionados aos idosos na literatura reflete as reais demandas que essa população poderá apresentar durante seu acompanhamento pelas equipes da ESF, e seu uso dará suporte à documentação sistemática do cuidado clínico de enfermagem, usando a CIPE como terminologia de referência. Portanto, revela-se como possibilidade de melhorias na prática clínica, mediante o aprimoramento das habilidades do enfermeiro na identificação, manejo e prevenção das alterações no idoso.

Em relação à categorização dos termos nos eixos, segundo o Modelo de Sete Eixos da CIPE versão $2013^{(14)}$, a predominância de termos alocados no eixo Foco - 138 termos constantes e 29 não constantes nessa terminologia - pode se justificar pelo fato de que o mesmo se refere à área de atenção relevante para a Enfermagem. Deste modo, reunirá um número substancial de termos, tendo em vista a complexidade e a especificidade do atendimento à pessoa idosa, que exige dos enfermeiros a capacitação para intervir preventiva e terapeuticamente, conforme as necessidades de cada indivíduo, considerando a amplitude do processo de envelhecimento humano ${ }^{(17)}$.

O fato de a maioria dos termos identificados ter sido classificada como constantes na CIPE ${ }^{\circ}$ assegura a confiabilidade dessa terminologia enquanto instrumento tecnológico para inserção em sistemas de informação e registro da prática clínica em âmbito mundial, com vistas ao desenvolvimento científico e tecnológico da profissão(18).

A validação de 61 termos considerados não constantes na CIPE também chama atenção neste estudo, e fornece indícios da utilização de uma linguagem própria na prática clínica de enfermagem com idosos na APS. Portanto, é necessário que os termos utilizados nesta área específica possam ser descritos e inseridos na $\mathrm{CIPE}^{\circ}$, garantindo o dinamismo e a contínua evolução dessa terminologia, conforme recomenda o $\mathrm{CIE}^{(19-}$ 20)

A etapa de validação dos termos identificados, por especialistas da área, constitui uma etapa de fundamental importância, pois garante a confiabilidade necessária para validar os elementos considerados relevantes para a prática clínica do enfermeiro, e sua posterior inclusão na CIPE .

Dessa forma, a dificuldade de captação de especialistas competentes para participar da etapa de validação dos termos pode guardar relação com o fato de que o debate acerca do uso da CIPE ${ }^{\bullet}$, enquanto 
terminologia de referência, no Brasil, ainda é recente e pouco difundido, apesar de ser evidente o crescimento do número de pesquisadores com interesse no assunto e preocupados com uma linguagem unificada para a profissão(16,21).

Acredita-se que construir um banco de termos para a prática clínica de enfermagem que atenda às necessidades do idoso e que seja utilizado de forma efetiva pelos enfermeiros atuantes na ESF ainda é um grande desafio. Contudo, espera-se que o banco de termos elaborado neste estudo possa constituir um produto tecnológico com possibilidade de uniformizar as práticas de enfermagem voltadas aos idosos atendidos nesse nível de atenção. Além disso, trata-se de etapa fundamental para a elaboração de enunciados de diagnósticos, resultados e intervenções de enfermagem para essa especialidade, que constituem os subconjuntos terminológicos, usados para apoiar e melhorar a prática clínica, a pesquisa e o ensino, favorecer a adoção de uma linguagem unificada e acessível aos enfermeiros em âmbito mundial e facilitar a tomada de decisões no cuidado clínico ao idoso que vive na comunidade; embora não substituam o julgamento clínico e a tomada de decisões pelo enfermeiro ${ }^{(4,14)}$.

\section{CONCLUSÃO}

Neste estudo foram identificados termos relevantes para a prática clínica de enfermagem com idosos comunitários, por meio da extração, uniformização e normatização de termos em documentos oficiais sobre idosos publicados no Brasil, os quais foram validados por especialistas competentes na área de Enfermagem e mapeados aos termos contidos na CIPE $^{\circledR}$ versão 2013, resultando em 271 termos constantes e 61 não constantes nessa terminologia. Isso demonstra que é possível a utilização desse sistema de classificação na prática clínica de enfermagem com idosos na ESF.

Esses termos serão utilizados para a construção de um subconjunto de enunciados de diagnósticos, resultados e intervenções de enfermagem, que servirá de base para o cuidado sistematizado do enfermeiro às pessoas idosas atendidas nesse nível de atenção.

Como principal limitação do estudo, destaca-se o fato de que o processo de extração e análise dos termos foi realizado por um único pesquisador, o que não impede que perdas relevantes tenham ocorrido, mesmo que seguidas as diretrizes propostas na literatura.

Acredita-se, contudo, que os resultados desta pesquisa trarão importantes contribuições para a prática clínica de enfermagem com idosos na atenção básica, ao propor a utilização da linguagem específica da profissão e a uniformização da comunicação entre os profissionais que atuam nesse nível de atenção, o que resultará em maior visibilidade, reconhecimento profissional e autonomia ao enfermeiro na ESF.

\section{REFERÊNCIAS}

1. Darder JJT, Carvalho ZMF. La interface del cuidado de enfermería con las políticas de atención al anciano. Rev Bras Enferm. 2012; 65(5):721-9.

2. Oliveira JCA, Tavares DMS. Elderly attention to health strategy in the family: action of nurses. Rev Esc Enferm USP. 2010; 44:774-81. 
3. Conselho Internacional de Enfermagem. Classificação Internacional para a Prática de Enfermagem - CIPE : versão 2. São Paulo: Algol Editora, 2011.

4. Clares JWB, Freitas MC, Guedes MVC, Nóbrega MML. Construction of terminology subsets: contributions to clinical nursing practice. Rev Esc Enferm USP. 2013;47(4):965-70.

5. Alves KYA, Dantas CN, Salvador PTCO, Dantas RAN. Vivenciando a Classificação Internacional de Práticas de Enfermagem em Saúde Coletiva relato de experiência. Esc Anna Nery. 2013; 17(2):381-8.

6. Marin HF. Vocabulário: recurso para a construção de base de dados em enfermagem. Acta Paul Enferm. 2000; 13(1):86-9.

7. Brasil. Lei n. 8.842, de 4 de janeiro de 1994: dispõe sobre a política nacional do idoso, cria o Conselho Nacional do Idoso e dá outras providências. Diário Oficial da União. Brasília, 1994.

8. Brasil. Lei n. 10.741, de 01 outubro de 2003: dispõe sobre o Estatuto do Idoso e dá outras providências. Diário Oficial da República Federativa do Brasil, 03 out 2003. Seção 1.

9. Ministério da Saúde (BR). Portaria nº 2.528, de 19 de outubro de 2006: dispõe sobre a política nacional de saúde da pessoa idosa. Diário Oficial da União. Brasília (DF); 2006.

10. Ministério da Saúde (BR). Secretaria de Atenção à Saúde. Departamento de Atenção Primária. Envelhecimento e saúde da pessoa idosa. Cadernos de Atenção Primária, n.19. Brasília (DF): Ministério da Saúde; 2007.

11. Ministério da Saúde (BR). Secretaria de Atenção à Saúde. Secretaria de Gestão do Trabalho e da Educação na Saúde. Guia prático do cuidador. Brasília: Ministério da Saúde; 2008.

12. Lynn MR. Determination and quantification of content validity. Nurs Res. 1986 Nov-Dec; 35(6):382-5.

13. Batista CG, Matos MA. O acordo entre observadores em situação de registro cursivo: definições e medidas. Psicologia. 1984; 10(3):57469.

14. International Council of Nurses. International Classification for Nursing Practice: ICNP ${ }^{\circledR}$. Version 2013. Geneva: ICN; 2013. [cited 2014 Nov 10]. Available from: http://www.icn.ch/pillarsprograms/icnpr-translations/.

15. Conselho Nacional de Saúde (BR). Resolução n.466, de 12 de dezembro de 2012. Brasília; 2012 [citado 2014 Nov

11]. Disponível em: http://www.conselho.saude.gov.br/web_comissoes/conep/index.html.

16. Clares JWB, Freitas MC, Guedes MVC. Methodological approach for the development of terminology subsets ICNP : an integrative review. Rev Esc Enferm USP. 2014; 48(6):1119-26.

17. Clares JWB, Freitas MC, Galiza FT, Almeida PC. Sleep and rest needs of seniors: a study grounded in the work of Henderson. Acta Paul Enferm. 2012; 25(esp.1):54-9.

18. Garcia TR, Nóbrega MML. A terminologia CIPE e a participação do Centro CIPE brasileiro em seu desenvolvimento e disseminação. Rev Bras Enferm. 2013; 66(esp):142-50.

19. International Council of Nurses. ICN Accredited Centres for ICNP Research \& Development. Geneva: International Council of Nurses; 2013.

20. Silva RS, Nóbrega MML, Medeiros ACT, Jesus NVA, Pereira A. Termos da CIPE empregados pela equipe de enfermagem na assistência à pessoa em cuidados paliativos. Rev Eletr Enf [Internet]. 2015 [cited 2015 set 15 ] 17(2):269-77. Available from: http://www.fen.ufg.br/revista/v17/n2/pdf/v17n2a11.pdf.

21. Barra DCC, Dal Sasso GTM. The nursing process according to the International Classification for Nursing Practice: an integrative review. Texto Contexto Enferm. 2012; 21(2):440-7. 